\title{
Sunflower oil as fuel in a diesel engine
}

\author{
C. Arapatsakos, A. Karkanis, D. Cristoforidis, D. Mitroulias \\ \& C. Teka \\ Mechanical Engineering Laboratory, Democritus University of Thrace, \\ Greece
}

\begin{abstract}
Nowadays the petroleum oil crisis is leading to the use of renewable sources of energy. This paper examines the use of diesel-sunflower oil mixtures in diesel engines. The mixtures used are the following: diesel-10\%sunflower oil, diesel-20\% sunflower oil, diesel-30\% sunflower oil, diesel- $40 \%$ sunflower oil, diesel-50\% sunflower oil.

For those mixtures the gas emissions of Carbon monoxide (CO), hydrocarbons (HC) and Nitrogen monoxide (NO) are being measured and the fuel consumption is also examined.
\end{abstract}

\section{Introduction}

One of the most important and renewable sources of energy is biomass. Biomass is every material that is produced from an embryo organism in to a renewable base, such as plants, animals and microorganisms. Biomass is a source of energy, which is continuously renewable due to photosynthesis. During photosynthesis, committed solar energy is changed into chemical (energy). During the combustion of biomass the committed solar energy is changed into thermic, while the dioxide of coal $\left(\mathrm{CO}_{2}\right)$ (which is committed for the production) returns into the atmosphere, while the inorganic elements that are contained in the ash enrich the soil with nutritious elements. Nowadays, the use of biomass covers approximately $4 \%$ of the total energy that is consumed in the USA and $45 \%$ of the renewable sources of energy [1]. It is important to mention that biomass constituted the first fuel used by human beings.

An important source of biomass is the forest. The kinds of biomass for energy that are produced in the forest are firewood and charcoals, as well as the 
remains of loggings and clearings of forests. It is essential that all of these be removed in order not to constitute fuel matter in forest fires. Other kinds of biomass that can be used for the production of energy are various materials of plant origin, such as agricultural remains (e.g. straw), sub products (e.g. fiery-woods), energy cultures, material of animal origin and remains from veterinary surgeon units as well as remains of fisheries and their sub products, urban waste etc.

All these kinds of biomass constitute an important renewable source of energy, which is produced in large quantities. Also the energy development of biomass may have, as a result, multiple environmental advantages.

Biomass can be used for the production of liquid fuel (called bio-fuel), which is used for transportation in many countries of Europe, USA etc. [2].

Bio-diesel is also produced from oily plants (soya, sunflower) animal greases, products of carcasses, and used oils.

\subsection{Advantages of biomass}

Some of the advantages of biomass that make it an attractive source of energy are the following:

1. The combustion of biomass has a null balance of dioxide of coal $\left(\mathrm{CO}_{2}\right)$ and does not contribute to the green house phenomenon, because the quantities of dioxide of coal $\left(\mathrm{CO}_{2}\right)$ that are released during the combustion of biomass are committed again by the plants for the creation of biomass.

2. The zero existence of sulphur in biomass contributes considerably to the restriction of emissions of dioxide of sulphur $\left(\mathrm{SO}_{2}\right)$, which is responsible for acid rain.

3. Since Biomass is a domestic source of energy, its exploitation of energy contributes considerably to the reduction of dependence on imported fuels, improvement of the commercial balance, in the guaranty of energy supply and in the saving of exchange.

4. The energy development of biomass in a region increases the number of people working in agriculture with the use of alternative cultures (several kinds of cane, sorghum), as well as the creation of alternative markets for the traditional crops (sunflowers etc.), and it also ensures people can sustain their homes in the agricultural area. Consequently it contributes to the socio-economic growth of the region. Studies have shown that the production of the liquid bio-fuels has positive results in the employment market in the agricultural and the industrial sectors. Bio-fuels are liquid or gas fuels that are produced from the biomass. Biomass can replace the conventional mineral fuels in engines, totally or partially.

\subsection{Advantages of bio-diesel}

Bio-diesel is methilester, which is produced mainly from oleaginous seeds (i.e. sunflower, etc.), it can be used either alone or mixed with diesel into petroleum engines. The main way of producing bio-diesel is the metesteropiosi of vegetable oils. Bio-diesel is a substitute for diesel from the raw diesel (in pure form or mixed), and it has the following advantages: 
1. It may decrease our dependence on the import of fuels. The sector of transports presents an important increase in the consumption of fuels, diesel and petroleum. Bio-diesel can be produced locally from agricultural production and from used oils of foods. With the possibility of direct use in the engines, it can contribute to the reduction of diesel demands from oil and accordingly in the corresponding reduction of the import of raw diesel.

2. It contributes to the reduction of the emissions of green house gases. In the previous century, the combustion of mineral fuels contributed dramatically to the increase in levels of dioxide of coal $\left(\mathrm{CO}_{2}\right)$ and other green house gases, which trap the heat in the atmosphere. The fact that bio-diesel is a totally renewable fuel may contribute to the reduction of emissions of green house gases in the transport sector.

3. It may be helpful in the reduction of atmospheric pollution and accordingly the danger to the public health. Relevant studies have shown that the use of bio-diesel can cause a reduction in the swing bodies (PM), carbon monoxide (CO), hydrocarbons ( $\mathrm{HC})$, dioxide of sulphur $\left(\mathrm{SO}_{2}\right)$, nitrogen oxides $(\mathrm{NOx})$ and other toxic air [3].

4. It can be helpful to the economy of the country. The reduction in imports of petroleum and the reformation of cultures contribute to an increase in the economy.

In May 2003, the European Committee adopted the directive 2003/30/EK[EK2003] [4], regarding the propulsion of bio-fuel or other renewable fuels used for the transportation [5-7].

Sunflower oil is an annual plant that belongs to the Compositae family. In Greece, it is mainly cultivated in the northeast part of the country as a source of vegetable oil for food.

The question that arises is how a four-stroke diesel engine behaves in terms of pollutants and operation, when it uses mixed diesel and sunflower fuel.

\section{Instrumentation and experimental results}

In the experimental stage sunflower oil in a mixture of diesel has been used directly in a four-stroke diesel engine and not elaborated in the form of bio-diesel. Specifically a diesel (diesel 25-5) mixture, diesel-10\% sunflower oil (H10), diesel-20\% sunflower oil (H20), diesel-30\% sunflower oil (H30), diesel-40\% sunflower oil (H40) and diesel-50\% sunflower oil (H50) have been used in a four-stroke Ruggerini type RD-80 diesel engine, with a volume of $377 \mathrm{cc}$, and power of $8.2 \mathrm{hp}$, which was connected to a water centrifugal pump. Measurements were made when the engine was moving at 1000, 1500, 2000 and 2500rpm.

\subsection{Experimental measurements}

During the experiments, the following measurement were taken:

- The percentage of CO

- The ppm (parts per million) of $\mathrm{HC}$

- The ppm (parts per million) of NO 
- The percentage of smoke

- The supply of water

- Fuel consumption

The measurement of rounds/min of the engine was made by a portable tachometer (digital photo/contact tachometer) named LTLutron DT-2236.

A specific measurement device named SMOKE MODULE EXHAUST GAS ANALYSER MOD 9010/M, which has been connected to a PC unit, measured smoke. A HORIBA Analyzer MEXA-324 GE measured the $\mathrm{CO}$ and $\mathrm{HC}$ emissions. A Single GAS Analyser SGA92-NO measured the NO emissions.

\subsection{Experimental results}

The experimental results are shown in figures 2-5.

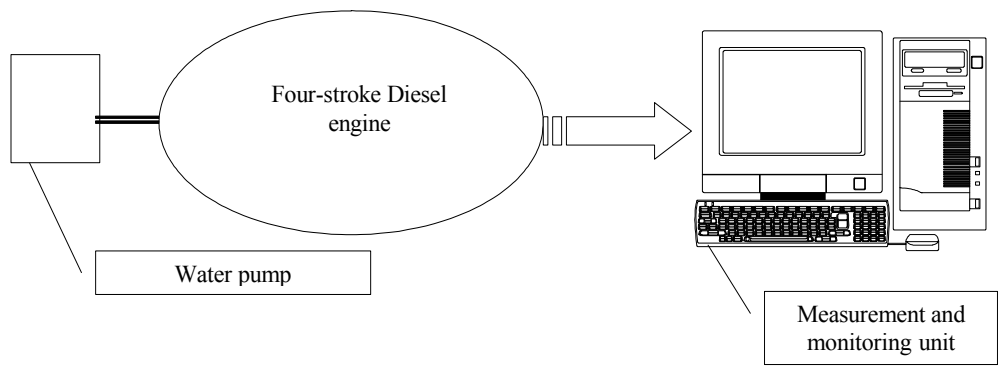

Figure 1: Experimental layout.

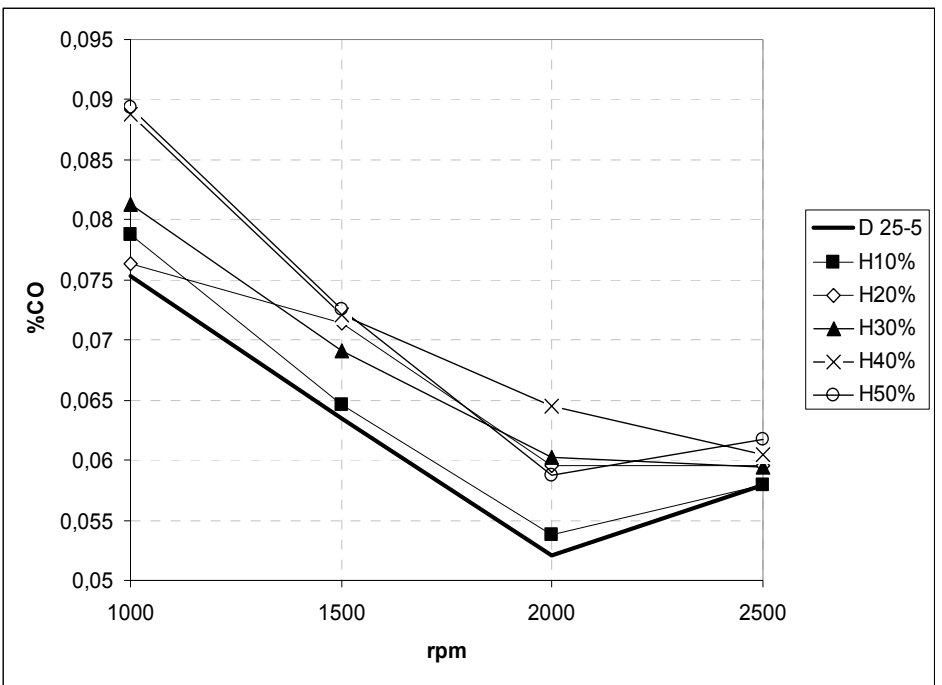

Figure 2: The $\mathrm{CO}$ variation at different rpms with different mixtures. 


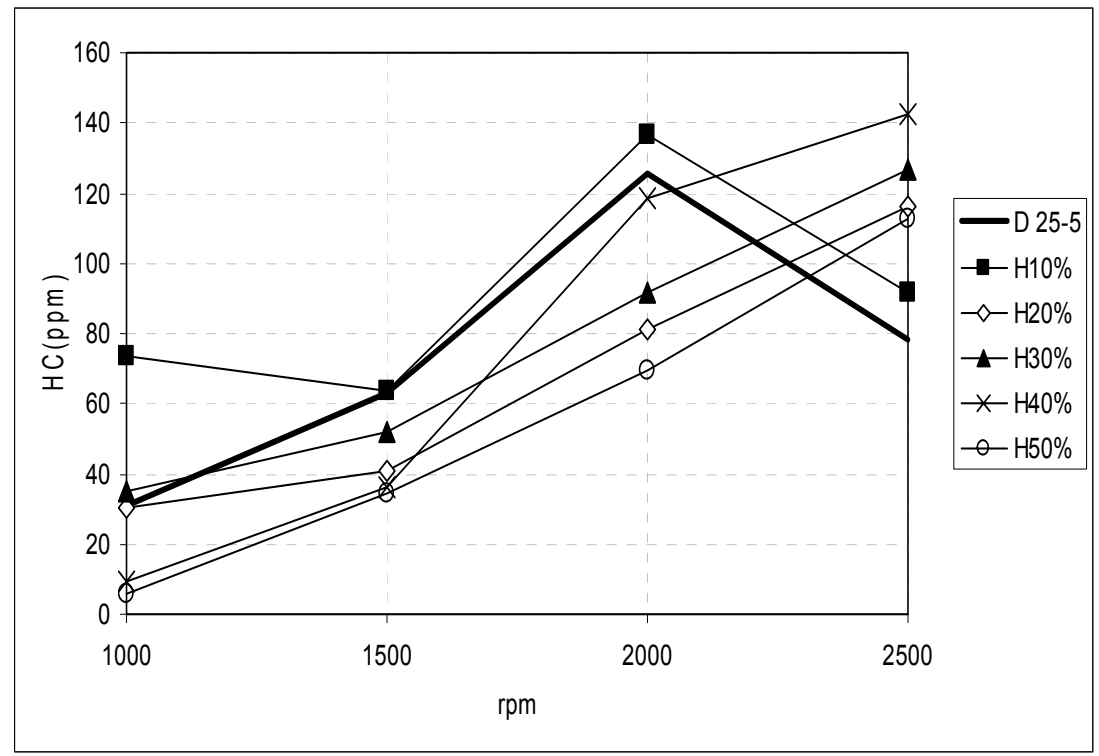

Figure 3: $\quad$ The $\mathrm{HC}$ variation at different rpms with different mixtures.

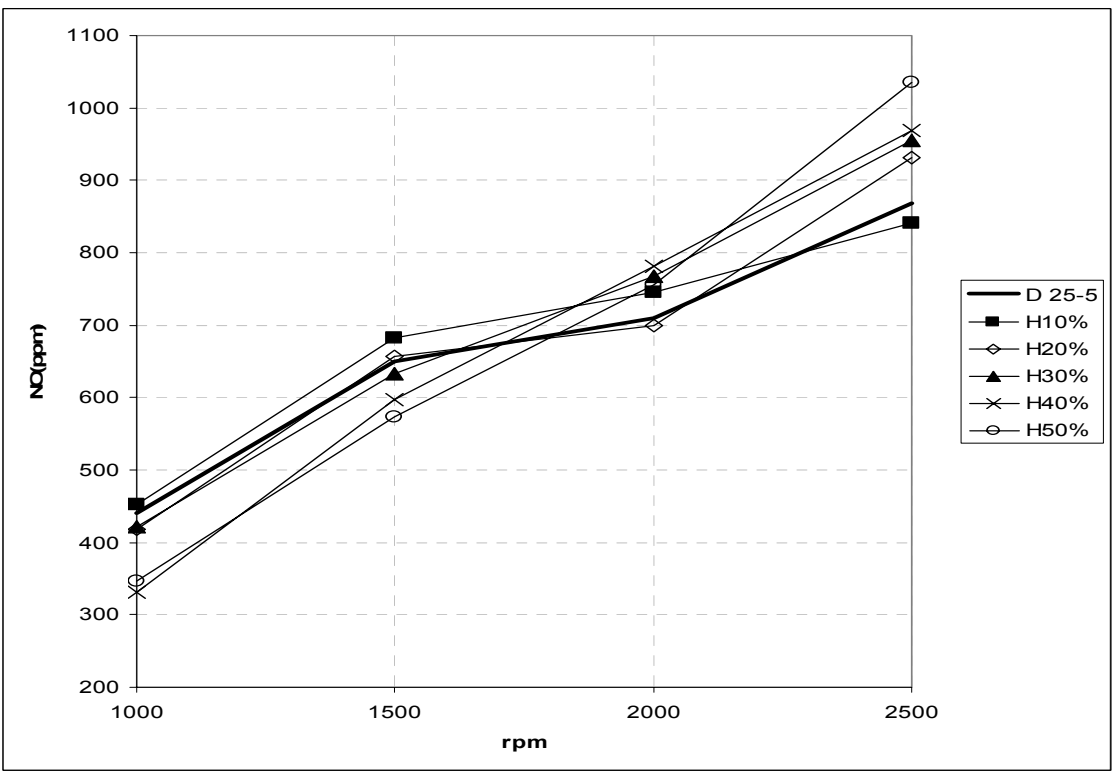

Figure 4: The NO variation at different rpms with different mixtures. 


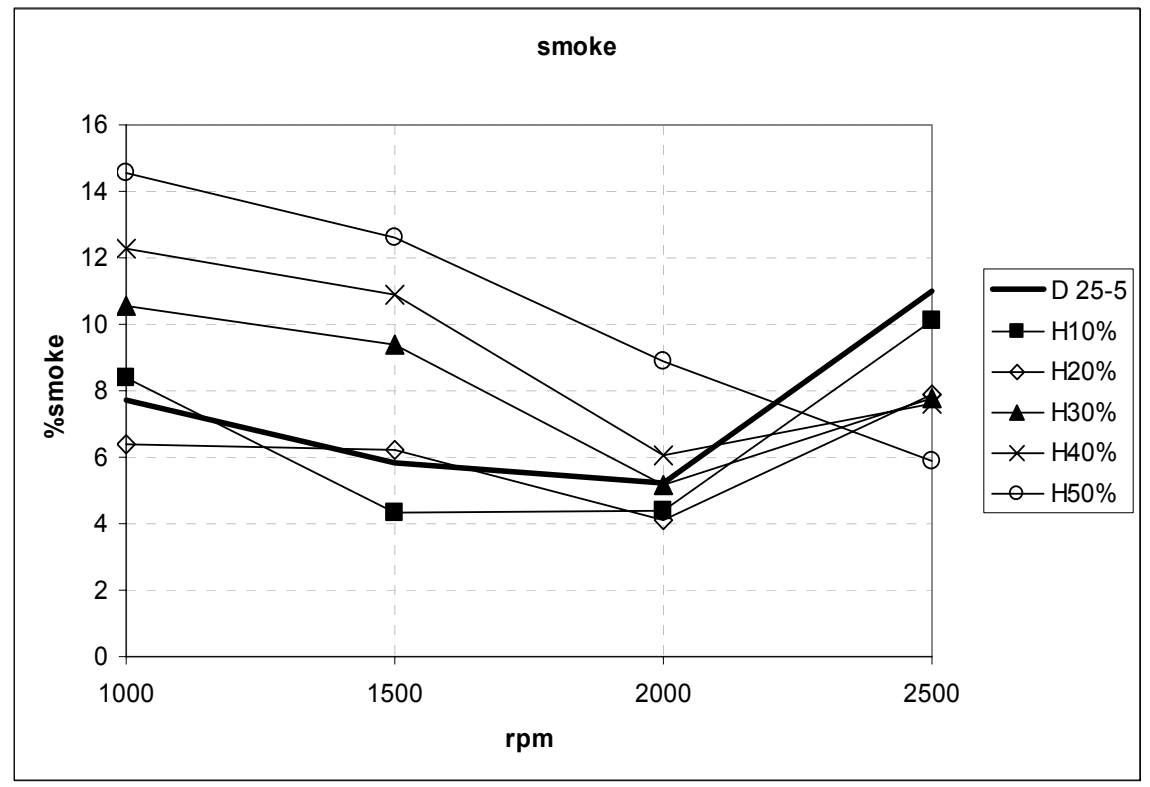

Figure 5: The smoke variation at different rpms with different mixtures.

From figures $2-5$ it is clear that the use of different mixtures can constitute changes to $\mathrm{CO}, \mathrm{HC}, \mathrm{NO}$, as well as smoke.

It is also important to note the fact that there were no changes in the turns of the engine, or in the supply of water for the use of the mixtures. Finally, as far as the consumption is concerned, changes did not exist with the use of different mixtures.

\section{Conclusion}

Regarding CO emissions, diesel presents the least concern of all the mixtures of diesel-sunflower oil. The diesel-sunflower oil mixtures (excluding H10\%), present lower $\mathrm{HC}$ emissions compared to diesel. When the engine works in low turns (rpm), diesel appears to have greater $\mathrm{NO}$ emissions than the diesel-sunflower oil mixtures. The situation is reversed when the engine works in higher turns (rpm). The $\mathrm{H} 10 \%$ and $\mathrm{H} 20 \%$ mixtures present less smoke emissions than diesel. The power of the engine has not been affected from the use of different mixtures, as there was no change in turns or water supply. The consumption of fuel has not been influenced by the use of different mixtures. Future research will constitute the use of percentages of sunflower oil under $10 \%$. $\mathrm{H} 10 \%$ and $\mathrm{H} 20 \%$ present better behavior with regard to pollution than all the other mixtures. The consumption also remains the same with diesel, even if the constitution of the fuel changes. 


\section{References}

[1] Handbook of Chemistry and physics, 62 ${ }^{\text {nd }}$ Edition, 1981, The Chemical Rubber Company Press, Inc.

[2] Owen K. and Coley T., Automotive Fuels Reference Book, SAE 1995.

[3] Schafer F. and Basshuysen van R., Reduced Emissions and Fuel Consumption in Automobile Engines, SAE 1995.

[4] Dr. Kiriakos Maniatis DG TREN Biofuels \& Energy "European Policies for Biofuels" 2005.

[5] Timothy T. Maxwell and Jesse C. Jones Alternative fuels: Emissions, Economics and Performance, SAE 1995.

[6] Keith Owen and Trevor Coley, Automotive Fuels Reference Book, SAE 1995.

[7] Arapatsakos C., Karkanis A., and Sparis P., issue 7, volume 2, July 2006, ISSN 1790-5079, Environmental Contribution of Gasoline - Ethanol Mixtures 